\title{
Élaboration d'un cadre d'analyse théorique de la contribution des activités économiques au développement territorial
}

\author{
Manu Tranquard ${ }^{\mathrm{a}}$
}

RÉSUMÉ. L'objectif de la présente contribution est de proposer une grille d'analyse théorique pour évaluer de quelle manière une activité économique peut contribuer au développement du territoire qui la porte. Plus spécifiquement, il s'agira d'identifier les approches qui proposent des modèles explicatifs du développement économique des régions, puis celles qui construisent, pour une région donnée (dépendant des facteurs de dotation en ressources, de la localisation, etc.), des modèles prescriptifs et pratiques de développement. Dans un premier temps, il s'agira de préciser quels liens entretiennent les notions de développement, de croissance, d'économie et d'espace, et d'évoquer selon une trajectoire historique le contexte de la territorialisation des approches développementales. Ce détour permettra de mieux saisir les conséquences de l'intégration du concept de territorialité dans la sphère économique. Dans un second temps seront identifiées, analysées et comparées les théories fondatrices du développement territorial. Le but consiste à produire un cadre d'analyse de la contribution des activités humaines au processus de développement.

\begin{abstract}
The objective of this contribution is to propose a theoretical analysis grid to assess how an economic activity can contribute to the development of the territory. More specifically, it will be a question of identifying the approaches which propose explanatory models of the economic development of the regions, then those which construct, for a given region (depending on factors of endowment in resources, location, etc.), prescriptive and practical models of development. Firstly, it will be a question of specifying which links maintain development, growth, economy and space, and of evoking, according to a historical trajectory, the context of the territorialization of the developmental approaches. This will allow to better understand the consequences of integrating the concept of territoriality into the economic sphere. Secondly, the founding theories of territorial development will be identified, analyzed and compared. The objective is to produce a framework for analyzing the contribution of human activities in the development process.
\end{abstract}

\section{Introduction}

Comment expliquer qu'une portion de territoire parvienne à tirer son épingle du jeu sur l'échiquier de l'économie mondiale, alors que son voisin immédiat connaît les pires difficultés pour surnager? Comment une même région peut-elle passer en quelques décennies de la croissance au déclin ou inversement? Cette double interrogation remet en question les mécanismes du développement selon deux paramètres : l'espace et le temps. Elle s'inscrit dans la logique qui, depuis son origine, fonde essentiellement l'économie régionale, où les approches théoriques ont pour la plupart tendu à proposer des modèles explicatifs du développement. «Exposer les raisons de la croissance régionale est l'un des objectifs privilégiés de l'économie régionale ", écrivait Aydalot (1985, p. 107). Cet objectif a tout à la fois une visée théorique et pratique. Il s'agit de comprendre les mécanismes en jeu et d'essayer par la suite d'approcher un modèle idéal de développement pour mieux le reproduire.

Dans cette logique, l'objectif de la présente contribution repose essentiellement sur une mise en perspective des principales théories du développement territorial et régional. Afin de mieux cerner

\footnotetext{
a Professeur, Unité d'enseignement en intervention plein air, Laboratoire d'expertise et de recherche en plein air (LERPA), Université du Québec à Chicoutimi
} 
les conséquences de l'intégration du concept de territorialité dans la sphère économique, il convient alors de préciser en premier lieu quels liens entretiennent développement, croissance, économie et espace, puis d'évoquer selon une trajectoire historique le contexte de la territorialisation des approches développementales.

\section{Prolégomènes à l'étude du développement économique territorial : mise en perspective des concepts}

\subsection{Le développement : survol d'un concept polymorphe}

Depuis son origine jusqu'à aujourd'hui, le concept de développement est demeuré par nature polymorphe. Bon nombre d'auteurs (Rist, 2007; Jean, 2008; Theys, 2002) ont en effet relevé que le sens qui lui est conféré varie selon le cadre des diverses sciences sociales ou autres dans lequel il peut être appréhendé - sans compter les variations au cours des siècles. Il est ainsi hasardeux d'en proposer une définition définitive; tout au plus, est-il possible d'en déterminer quelques éléments constitutifs.

Au regard des définitions les plus usuelles, le développement est un phénomène global qui correspond à l'amélioration qualitative et durable d'une société et de son fonctionnement. Dans son acception la plus large, et notamment pour les instances internationales, le développement est le développement humain, et il est synonyme de progrès en extension ou en qualité. Il s'agit d'un "mouvement vers le haut de tout le corps social» (Myrdal, cité par Brasseul, 2008, p. 32), ou encore de l'ensemble des transformations institutionnelles, psychosociales, mentales qui permettent une croissance durable, une diminution des inégalités, le maintien des cultures particulières, en symbiose avec l'environnement (Austry, Caire, Leduc et Lebret, 1972).

Selon le Programme des Nations unies pour le développement (PNUD, 1991), le développement humain présente plus précisément deux aspects : d'une part, la création de capacités personnelles (par les progrès obtenus en matière de santé, de savoir et d'aptitudes) et, d'autre part, l'emploi que les individus font de ces capacités dans leurs loisirs, à des fins productives ou culturelles, sociales et politiques.

Sous ce jour, le développement s'apparente au changement social, qui découle du processus économique, du système politique, mais aussi de l'évolution des mentalités, des rapports à l'autre et de la perception de la nature (Rist, 2015). À ce titre, le développement nous projette hors du domaine de l'économie stricto sensu et oblige à considérer de nouvelles dimensions.

Toutefois, traditionnellement, les transformations sociales apparaissent principalement comme les résultantes de la croissance de la production et des transformations des infrastructures, lesquelles créeraient un terreau économique fertile au développement humain. Il y aurait, toujours selon cette idéologie traditionnelle, une primauté de l'économie comme soutien au développement: "Le développement économique est celui qui, par le biais du renforcement des moyens de production et de la croissance, augmente le revenu des individus, hommes et femmes, leur accès non discriminatoire à l'activité économique, leur capacité de satisfaire leurs besoins de base et le respect dont ils jouissent dans leur communauté » (Martens, 2005, p. 23).

Or, la place de l'économie dans le processus de développement humain fait globalement toujours débat: bougie d'allumage, vecteur unique ou support principal? Afin d'appréhender davantage le phénomène, il convient d'apprécier de manière liminaire les principales facettes théoriques du développement au sens large, dont on comprend qu'il est un concept polymorphe ou multifacette qui intègre a priori des éléments de nature purement économique (la croissance, l'expansion), des composantes individuelles et sociales (l'autonomie, l'émancipation ou encore, en creux, la lutte contre le sous-développement) et notamment des éléments techniques (le progrès et l'innovation).

\subsubsection{Le développement lato sensu comme processus progressif et continu}

Au sens de la définition du PNUD (1991) et dans la lignée des travaux de Perroux (1955), le développement s'appuie initialement sur l'amélioration des capacités humaines - meilleure satisfaction des 
besoins fondamentaux (alimentation, santé, éducation); réduction des inégalités, du chômage et de la pauvreté -, laquelle crée des conditions d'accroissement de la productivité. "Les premières conditions d'une production élevée sont que les masses populaires soient alphabétisées, en bonne santé et suffisamment bien nourries" (Viner, cité par Brasseul, 2008, p. 12).

Pour d'autres auteurs, la croissance économique est une condition nécessaire du développement puisqu'elle seule permettra d'améliorer la qualité de vie, d'augmenter "l'étendue des choix humains (Lewis), de dégager des ressources en faveur de la santé, de l'éducation, de l'environnement, et d'accroître l'indépendance économique nationale, en rendant l'aide étrangère moins nécessaire » (Brasseul, 2008, p. 23). Le cumul des approches permet de dessiner les contours d'un cercle vertueux où accessibilité aux ressources essentielles, progrès technique et croissance économique alimentent conjointement le développement (voir lien de « causalité circulaire » selon Myrdal, 1957).

En ce sens, un premier principe est que le développement est un phénomène dynamique et qu'il n'existe pas, pour une nation donnée, de « richesse spontanée ». Quelle que soit la voie empruntée, des liens de causalité sont à rechercher entre ressources naturelles (entendues au sens large) et valorisation de celles-ci jusqu'à production de richesses. Tendre vers cet objectif d'optimisation des ressources fonde la quête du développement. C'est la « directionnalité » du développement dont parle Rist (2007), par analogie avec la croissance des végétaux, dont la graine se transforme en arbre selon un processus continu et systématique.

Certes, déterminer quel est le bras de levier le plus efficace pour stimuler le développement est affaire de débat. Or, il semble convenu que la mécanique du développement soit notamment enclenchée "dès lors qu'un surplus apparait (la production dépasse la consommation) et qu'il est affecté à l'accroissement de la capacité de production» (Brasseul, 2008, p. 103). Cette approche rejoint celle de l'économiste classique Adam Smith (1776), selon qui «l'enrichissement des nations est lié à l'accumulation du capital par une certaine partie de la société. S’il est réinvesti, le surplus économique permet un accroissement de la production, grâce notamment à la division du travail et au progrès technique » (Vivien, 2003, p. 3). Le développement passerait donc nécessairement par l'économie.

Cette vision d'une progressivité du développement a été notamment explicitée par l'économiste Walt Whitman Rostow (1962) dans l'objectif d'appliquer la théorie économique à l'analyse de l'histoire économique. Pour cet auteur, l'essentiel de la dynamique du développement tient à la modification des comportements d'épargne, qui permettront un accroissement de l'investissement et, donc, une accélération de la croissance de la production par tête: passage de la stagnation (étape 1) à une accumulation primitive (étape 2) qui financera le décollage ou take-off (étape 3). La perspective de Rostow est fortement influencée par l'analyse néoclassique de la croissance puisque l'épargne est le point de départ du processus de croissance économique, puis du développement humain. Cette dynamique suppose une modification des conditions économiques et techniques ainsi que des mentalités.

\subsubsection{Le développement comme processus cumulatif complexe}

Dire que le processus de développement repose sur le principe du cumul signifie deux choses. La première est que le développement procède de l'addition; la seconde, qu'il provient également d'un processus de superposition.

Selon divers auteurs (Proulx, 2008a, 2008b; Myrdal, 1957), le développement n'est pas qu'économique; il est en fait la résultante d'un cumul de capitaux variés: capital physique (infrastructures), économique et financier (fonds, épargne), humain (éducation et formation), social (solidarité) et de créativité (innovation). L'addition de ces capitaux sur un territoire donné, à un moment précis, permet d'induire le progrès social et la croissance économique. "Le développement commence à certains endroits mieux dotés en ressources et en compétences, c'est-à-dire en capacités à produire en fonction de la demande. Le cumul des facteurs étant alimenté par les effets positifs du développement (emplois, salaires, épargnes, investissement, production), des effets entraînants se produisent selon une démarche cumulative de cause à effet que Myrdal nomme la causalité circulaire » (Proulx, 2008a, p. 203). 
En conséquence, il existe un seuil en deçà duquel des facteurs pris isolément ne peuvent amorcer le développement. Et l'effet cumulatif ou boule de neige toucherait autant le développement que le sous-développement (voir processus dichotomique du développement selon Proulx, 2008a).

La notion de superposition réfère, quant à elle, au fait que le cumul de capitaux divers ne s'opérerait pas de manière anarchique, mais plutôt que le développement serait d'autant plus marqué que les facteurs se succèderaient dans un ordre préétabli. On rejoint ici la notion de progressivité du développement. Établir cette chaîne de développement relève des diverses théories du développement.

Un changement de perspective doit toutefois être introduit dès ce stade. L'économiste autrichien Joseph Schumpeter (1912) énonçait : «Additionnez autant de diligences que vous voulez, vous n'obtiendrez jamais une locomotive ». Ce faisant, il faisait une distinction entre les changements quantitatifs et qualitatifs que connaissent les économies modernes. La croissance est alors définie comme un changement quantitatif, tandis que le développement l'est par un changement qualitatif. Dans cette perspective, énoncer que le développement est un processus cumulatif ne peut être interprété que comme l'aveu de la complexité du processus, qui dépasse de loin de simples mécanismes d'amalgame.

\subsubsection{Le développement comme processus transversal}

Une étude de l'Institut national de la recherche scientifique - Urbanisation, Culture et Société (Brunelle et Shearmur, 2007) a présenté les systèmes d'indicateurs utilisés dans des juridictions comparables au Québec pour appréhender le développement des régions, voire le prévoir. Le but de cette analyse était de "proposer un cadre comparatif qui favoriserait l'étalonnage en matière d'indicateurs de développement économique» (p. 5). Au terme de cette étude, les auteurs ont affirmé qu'il est possible de structurer le développement selon quatre grandes dimensions : 1) population et migration, 2) bien-être et équité sociale, 3) environnement et durabilité ainsi que 4) structures économiques et performance.

Un exercice effectué par l'Institut de la statistique du Québec (2007) fait également ressortir quatre grandes dimensions du développement économique régional: 1) croissance, 2) développement socioéconomique, 3) innovation et 4) ressources humaines.

Une synthèse des deux sources précédentes nous permet d'établir quatre principaux facteurs contributifs du développement (voir figure 1). Ces quatre facteurs deviennent alors de facto autant d'indices pour mieux appréhender ce concept.

\begin{tabular}{c|c}
$\begin{array}{c}\text { Performances des structures économiques } \\
\text { (croissance) et des facteurs techniques de pro- } \\
\text { duction (innovation) }\end{array}$ & Qualité de vie et bien-être socioéconomique \\
\hline $\begin{array}{c}\text { Dynamique des populations } \\
\text { et profil des ressources humaines }\end{array}$ & Gestion durable de l'environnement
\end{tabular}

Figure 1 - Principaux facteurs contributifs du développement économique régional

\subsubsection{Le développement comme processus lié à la croissance économique}

Au terme de cette analyse liminaire, nous constatons que, si le développement est un processus global, il existe un consensus à lui faire inclure nécessairement une large part de développement économique. Bien plus, il semble que le développement humain (autonomie individuelle) et le développement collectif (entendu au sens de progrès social) ne sont envisageables que grâce au gain d'autonomie économique, sinon comme préalable; du moins, comme condition essentielle. L'économique conditionnerait le développement.

Ce gain d'autonomie économique s'appuie notamment sur la croissance économique. Et le principe du 
surplus semble jouer un rôle central dans le processus. Or, au-delà, quels liens existe-t-il alors entre croissance et développement économique? L'étroite imbrication qui les unit nous conduit à devoir souligner leurs spécificités et nous invite surtout à nous interroger sur les mécanismes qui permettent de passer de l'un à l'autre.

Pendant longtemps, les premiers travaux des pionniers du développement en témoignent, le développement a signifié obligatoirement l'obtention d'une croissance économique sur une longue période. Inversement, toute croissance créerait une amélioration du bien-être de la population et une diminution de la pauvreté. Les travaux s'intéressaient donc aux déterminants de la croissance. Plus globalement, la théorie libérale néoclassique dominante fondait alors la majorité des réflexions concernant les règles, méthodes et options de développement. Pour les tenants de cette théorie, le développement ne peut être qu'économique et, de surcroit, ne doit s'inscrire que dans la mise en œuvre du modèle occidental dominant.

En 1962, cependant, U Thant, secrétaire général des Nations unies, déclarait : «Le développement n'est pas simplement synonyme de croissance économique; c'est une croissance économique accompagnée de transformations » (Thant, 1962, p. 76). Thant créait ainsi une brèche dans la vision économiciste du développement. Plus récemment, des économistes du développement (Comeliau, 2006 Assidon, 1992; Hugon, 1991) soulignent les faiblesses structurelles de cette théorie dominante. Le développement ne peut se faire sans croissance, mais une politique de développement doit s'attaquer également à réduire les inégalités, à satisfaire les besoins fondamentaux, etc. Selon ces auteurs, les études de développement sont au carrefour de deux principales contradictions :

1. Elles sont souvent marquées par une dépendance idéologique à l'égard du modèle occidental dominant (et, en ce sens, ne devraient pas avoir vocation à l'universalité), ce que Hirschman (1984) nomme le «mono-économisme»;

2. Elles sont traditionnellement dominées par l'approche économique, alors que le développement est global.

Ainsi, selon Assidon (1992), «les théories du développement se sont affirmées comme un corpus distinct dans la science économique dès lors qu'elles ont postulé l'existence de spécificités communes à un ensemble de pays, en même temps qu'elles ont adopté l'idée que le développement ne se réduisait pas à la croissance » (p. 5).

Appréhender le développement uniquement au regard des théories économiques serait en ce sens réducteur. Depuis Perroux (1961) surtout, les économistes essaient en effet de faire la distinction entre la croissance et le développement économique en soulignant que le développement est une catégorie économique plus large que la croissance économique. «Au fond de cette différenciation se trouve la conviction que le développement économique a certains attributs qualitatifs (les changements des systèmes juridico-politiques, les changements qualitatifs non mesurables qui ont un impact sur la croissance économique, mais surtout sur la qualité de la vie dans un certain pays)» (Malaga, 2009, p. 3). Ce qui est reproché, c'est donc le réductionnisme économique dans l'approche du développement.

Le principe de la distinction des deux notions repose sur le fait que le concept de développement s'est imposé dans les analyses économiques en raison de la notion de croissance économique, eu égard à la situation particulière de certains pays ou régions du monde. En effet, selon le PNUD (1991) :

«- Bon nombre de pays en développement à forte croissance constatent qu'en dépit d'un taux de croissance élevé du PNB, ils n'ont pu réduire le dénuement socio-économique de larges groupes de leur population;

- Les nations industrialisées elles-mêmes comprennent peu à peu qu'un revenu élevé ne constitue pas une protection contre la montée rapide de problèmes tels que la toxicomanie, l'alcoolisme, le sida, les sans-abri, la violence et l'effondrement des relations familiales;

- Dans le même temps, quelques pays à faible revenu ont prouvé qu'il était possible d'atteindre des niveaux élevés de développement humain en utilisant habilement les moyens disponibles pour droits humains fondamentaux » (p. 10).

Du principe de distinction stricte entre croissance et développement (D. Hunt (1989) de l'University of Sussex parle de "paradigme structuraliste ») nait un certain nombre de constats sur leurs caractéristiques intrinsèques, qu'il est envisageable selon nous de synthétiser ainsi : 


\begin{tabular}{|ll|}
\hline Croissance & Développement \\
\hline Économique stricte & Global : économique, social, politique, culturel, etc. \\
Sectorisée & Généralisé (à tout le moins une moyenne) \\
Dimension corporative & Dimension collective \\
Évolution quantitative & Évolution qualitative \\
Pas de changements structurels & Changements structurels \\
Suivie à moyen terme & Perceptible uniquement à long terme \\
Ahistorique & Historiquement situé \\
S’exprime dans l'augmentation de la capacité de production & S’exprime dans la bonification des facteurs de production \\
Aspatiale & Spatialisé \\
Transculturelle & Culturel \\
N'implique pas nécessairement le développement & Présuppose notamment la croissance économique \\
\hline
\end{tabular}

Tableau 1 - Caractéristiques comparatives de la croissance et du développement Source : compilation de l'auteur

En théorie, il y a donc bien dichotomie marquée entre les deux concepts. Cependant, un survol historique des pratiques du développement invite à tempérer cette conclusion. Ainsi, 30 ans après le discours de U Thant, le PNUD (1991) affirmait : « De même que la croissance économique est nécessaire au développement humain, le développement humain est essentiel à la croissance économique» (p. 2). Et dans son édition de 1992 : «La question n’est pas de savoir quel doit être le volume de la croissance, mais quel genre de croissance rechercher» (PNUD, 1992, p. 3). Ce qui fait dire à Rist (2015) : «Il est aisé de constater que, dans la pratique, c'est bien la croissance économique qui est poursuivie, au nom du développement. Et de repenser au cercle vertueux et au principe de causalité circulaire proposé par Myrdal » (p. 309).

Cette mise en perspective étant faite, comment enclencher concrètement le processus de développement humain? Quels bras de levier actionner pour stimuler la croissance économique et, concomitamment, générer des changements structurels et un progrès social durable? $\mathrm{Il}$ apparait ainsi que toute analyse des processus de développement d'un territoire devrait se fonder initialement sur l'étude des modalités de la croissance économique, tout en portant sur celle des aspects sociaux structurels (politique, culturel, etc.). Il s'agit d'une démarche holistique qui souligne le caractère incontournable de l'économie comme bras de levier principal du développement. Et ce processus est clairement spatialisé.

\subsection{La spatialisation du développement humain}

Tout comme le développement est historiquement situé, il est spatialement localisé. Et le territoire apparait, en tant qu'espace construit par les acteurs et leurs réseaux, comme un ensemble organique à même d'embrasser le processus de développement dans sa complexité; il est la «scène» du développement (Klein, 2008). Ainsi, selon l'économiste Marc-Urbain Proulx (2008a) : « Le territoire n'est pas neutre dans le déploiement des activités économiques et sociales. Le territoire n'a pas qu'un contenu statique en matière de ressources naturelles, de bassin de main-d'œuvre, de marchés, de fournisseurs d'intrants, de concurrents, de concentrations humaines, de centres urbains, etc. Il possède aussi un contenu dynamique. Dynamisme qui n'est pas nécessairement inné, mais plutôt appris au fil du temps selon des circonstances encore mal comprises » (p. 256).

\subsubsection{Le contexte de la territorialisation des approches développementales}

D’un point de vue théorique, l'intérêt porté au territoire repose essentiellement sur la rupture paradigmatique vis-à-vis de la doctrine keynésienne dominante dans les années 1970 en matière de développement (Kaldor, 1957). Proulx (2008b) explique cette rupture par « la déception générale à l'égard des retombées réelles générées par les interventions exogènes largement effectuées depuis le début des années 1950 pour lancer la croissance économique selon une finalité de convergence dans le niveau mesurable du développement à travers l'espace » (p. 1).

Globalement, trois raisons semblent expliquer la spatialisation du développement et la convergence des théories du développement vers le territoire comme pierre angulaire de l'analyse et de l'intervention. La première raison tient au constat de la «non-reproductibilité des expériences historiques de développement » (Jean, 2008, p. 293), notamment des stratégies de développement économique du Nord dans le contexte des pays du Sud. Si le développement 
était un processus généralisé et relevait d'une dynamique globale ou à tout le moins générique et reproductible, la spatialisation du mécanisme ne poserait pas de questions. Or, l'existence même des disparités nord-sud annule cette prémisse. «La recette qui a si bien réussi en Europe de l'Ouest ne peut plus, deux siècles après, servir de modèle pour planifier le développement de l'Afrique sud-saharienne » (Jean, 2008, p. 293). Ainsi, le poids de la dotation en ressources n'est plus aussi prépondérant qu'il le fut dans le passé au regard de l'abaissement des coûts de transport, qui permet de s'approvisionner facilement à l'extérieur.

Historiquement, ce n'est qu'à partir des années 1950 que des économistes (Rosenstein-Rodan, Hirschman, Lewis, Nurkse, Myrdal et Prebischv) cherchent à développer des outils propres aux pays en développement. On trouve certes des fondements de l'économie du développement dans la pensée économique classique (Malthus, Ricardo). Cependant, l'économie du développement ne s'est affirmée comme étant une branche distincte de la science économique qu'avec l'amorce du processus de décolonisation. C'est d'ailleurs par la voie de la spatialisation que l'autonomie des théories du développement par rapport aux théories économiques classiques s'est réalisée.

Deuxième raison, qui découle de la première: à l'échelle intranationale, l'existence de déséquilibres spatiaux, abondamment soulignée notamment par Perroux (1955), illustre l'hétérogénéité des territoires : «La croissance n'apparait pas partout à la fois; elle se manifeste en des points ou des pôles de croissance, avec des intensités variables; elle se répand par divers canaux et avec des effets terminaux variables pour l'ensemble de l'économie » (p. 309). Cela malgré des décennies d'intervention exogène et des volontés étatiques de permettre une compétitivité équilibrée des agents économiques (Dugas, 1994) ainsi que des volontés politiques marquées d'aménager pour effacer les disparités. La persistance de dynamiques territoriales spécifiques semble renforcer la logique d'intégrer l'environnement local comme espace du développement.

Troisième raison: dans un contexte de mondialisation, l'émergence de projets de territoire, d'initiatives d'accroissement de l'autonomie ou de renforcement des pouvoirs locaux s'inscrit clairement dans une logique de processus social territorialisé. Le territoire devient le nouveau référent comme axe et soutien au développement, dans une perspective transversale.
Dans cette logique, et selon la notion du développement endogène (Garofoli, 1994), le territoire est à la base du développement; il s'incarne dans un espace particulier et y prend sa source. Il est le fruit de chacune des composantes territoriales d'un espace, c'est-à-dire les composantes naturelles, culturelles, économiques et sociales (Chabault, 2006).

\subsubsection{Les conséquences génériques de l'intégration du concept de territorialité}

Depuis que le territoire semble s'être inscrit comme une échelle de développement à privilégier, deux principales tendances se sont matérialisées, lesquelles peuvent être présentées à grands traits.

La première tendance concerne la spécialisation et la polarisation. La polarisation économique comme modèle de développement repose essentiellement sur la pensée de John Maynard Keynes (1936) et ses applications sur l'analyse spatiale qu'en a faite Perroux (1955). Dans la vision perrouxienne, la croissance doit être spatialement concentrée pour être intensive, toute diffusion spatiale n'étant qu'une conséquence postérieure de la polarisation. La théorie des pôles de croissance embrasse ce principe : la croissance doit être concentrée pour s'optimiser. Dans la même lignée, on observe ainsi depuis quelques années une multiplication des applications empiriques de ce concept: districts industriels, systèmes industriels localisés, complexes industriels localisés, pôles industriels, technopôles, parcs d'activités et, plus récemment, pôles de compétitivité. L'intérêt pour ces phénomènes de concentration géographique d'entreprises s'est d'ailleurs largement accentué depuis le succès de la Silicon Valley aux États-Unis. Ces différentes configurations peuvent être regroupées sous le nom générique de "système territorial de production», que Maillat (2003) définit comme «un ensemble caractérisé par la proximité d'unités productives au sens large (entreprises industrielles et de services, centres de recherche et de formation, organismes d'interface, organisations professionnelles, etc.), qui entretiennent des rapports marchands et non marchands d'intensité plus ou moins forte (Gilly, 1987) et qui génèrent une dynamique productive d'ensemble » (Maillat, 2003, cité par Gilly et Grossetti, 1993, p. 453).

En schématisant ce principe, la polarisation confère au territoire une fonction de creuset, de réceptacle au 
capital. Les capitaux physiques, humains, de créativité, etc. s'y cumulent. Ce cumul contribue à la richesse et au développement, et le territoire en est le siège.

Par ailleurs, selon les économistes Prager et Thisse (2009), des travaux récents tendent plutôt à " privilégier les notions de qualité et de variété des produits comme facteurs de compétitivité internationale, de préférence à celle de spécialisation sectorielle. Cette nouvelle forme de spécialisation porte sur les gammes et la qualité des produits à l'intérieur des secteurs d'activité plutôt que sur la nature des produits (Hummels et Klenow, 2005; Schott, 2004) » (p. 6). Pour les pays avancés, le facteur clé de la croissance résiderait donc davantage dans la capacité à produire des biens et services de haute qualité dans un ensemble diversifié de branches d'activité. Autrement dit, il faudrait privilégier la spécialisation fonctionnelle au détriment de la spécialisation sectorielle (Duranton et Puga, 2005).

La seconde tendance est donc celle de la diversification. Celle-ci rejoint les arguments développés il y a près de 60 ans par l'urbaniste Jane Jacobs (1961), qui mettait l'accent sur la diversité des activités comme facteur majeur de la dynamique des grandes métropoles. Cette diversité favoriserait le jeu des externalités intersectorielles et la capacité d'adaptation aux évolutions économiques. Elle permettrait également une meilleure résistance aux chocs sectoriels négatifs, facteur particulièrement important dans les économies où les ménages éprouvent de grandes difficultés à stabiliser leur consommation (Klibanoff et Morduch, 1995).

En ce sens, la multifonctionnalité des territoires concourrait également à leur développement. C'est sur le plan de la complémentarité et de l'imbrication des activités humaines - et notamment économiques - que se créerait le terreau fertile au développement entendu au sens large. Le territoire, en tant que forme particulière d'organisation, sert d'assise au développement.

Cette « double postulation » de polarisation/diversification s'intègre en large part aux principales approches théoriques qui ont jalonné la quête d'un modèle prescriptif ou, à tout le moins, explicatif de développement des territoires et des régions. Elle ne contribue en effet pas uniquement à intégrer les théories de la localisation des activités économiques, l'organisation et la structuration de l'espace ainsi que les interactions spatiales, mais s'inscrit aussi dans le cadre d'analyse des théories du développement régional.

\section{2. Étude croisée des théories du développement territorial : vers l'élaboration d'un cadre d'analyse actualisé}

Quels sont les principaux modèles explicatifs du développement territorial et régional? Sur quels principes reposent-ils? Permettent-ils, une fois mis en perspective, d'établir un cadre générique d'analyse du potentiel de contribution d'une activité économique au développement de la région où elle se déploie? Tenter de répondre à ces questions impose d'en appeler à l'histoire des théories économiques comme toile de fond, mais commande surtout de relever au sein de celles-ci les critères pratiques de participation au développement économique et les déterminants de la croissance.

\subsection{Les spécificités des approches économiques du développement au regard des déterminants de la croissance}

\subsubsection{Des théories classiques du développement au modèle keynésien de la croissance économique}

Les économistes de l'école classique ${ }^{1}$ ont instauré les premières méthodes d'analyse de la croissance pour rechercher les causes de l'enrichissement « des nations ». Leurs analyses interrogeaient les forces qui gouvernent le développement de l'activité économique. Il s'agissait alors essentiellement de dégager les tendances à long terme d'un tel développement et de déterminer de quelle manière asseoir l'action politique pour favoriser le progrès.

Leurs principales contributions se fondent sur le constat selon lequel le progrès repose directement sur le développement matériel de la société. Les forces qui y contribuent sont le progrès technique et la croissance de la population (qui accroît la quantité de travail disponible). La tendance à long terme qui est alors perçue est celle de la marche vers l'état stationnaire. L'action politique à mettre en œuvre pour favoriser le progrès technique est de mettre fin aux privilèges et aux monopoles.

Outre l'accroissement des moyens de production, Smith (1776) privilégie la division du travail, la spécialisation et le développement des échanges. 
Ces facteurs sont considérés comme exogènes, c'est-à-dire indépendants les uns des autres et extérieurs à la logique des agents économiques. Les théories classiques privilégient l'accumulation du capital, source d'investissement.

Toujours selon Smith (1776), la combinaison du travail et du capital est à la source du développement. L'enrichissement des nations est lié à l'accumulation du capital par une certaine partie de la société. S’il est réinvesti, le surplus économique permet un accroissement de la production, grâce notamment à la division du travail et au progrès technique. Il y a donc une relation entre la croissance économique et la dynamique des revenus. Par ailleurs, la division du travail étant d'autant plus grande que le marché sera étendu, les économistes de l'école classique soulignent le bénéfice des grands espaces économiques sur la croissance. Par extension, l'échange est ce qui favorise la création de richesses. Ainsi, pour générer la création de richesses, il faut favoriser l'échange, d'où le libéralisme.

Alors que l'école classique, et même par la suite les socialistes, cherchait à découvrir la vérité économique, l'analyse de Keynes (1936) est un schéma d'action qui préconise des mesures pour stimuler la consommation ou attirer l'investissement afin de «soutenir le système de production tout en générant des revenus dans les circuits économiques » (Livre XIII). Pour Keynes, c'est en effet la faiblesse de la demande globale (consommation, investissement, dépenses publiques, demande étrangère) qui, en déterminant le niveau d'emploi, génère la stagnation. A contrario, la croissance s'acquiert par une politique de relance de la demande et des dépenses publiques ainsi que par l'investissement. Dans cette perspective, le développement est d'autant plus efficace que la consommation est forte (le multiplicateur dépendant de la propension à consommer), donc que l'épargne est faible. À la différence des économistes de l'école classique, pour qui la recherche de l'équilibre économique et du développement passe par le trinôme offredemande-prix, l'analyse keynésienne s'appuie sur le trio épargne-investissement-revenu.

\subsubsection{Les analyses \\ factorielles de la croissance}

\section{Croissance économique et conditions d'accumulation}

\section{Les analyses postkeynésiennes de la croissance: le modèle de Harrod-Domar}

Au tournant des années 1940, au moment où triomphe la révolution keynésienne, Harrod (1939) et Domar (1946) entreprennent d'appliquer la théorie de Keynes à la croissance et élaborent un modèle de croissance selon lequel la croissance économique dans les économies de marché est instable. Leur modèle est en général considéré comme « le » modèle de croissance keynésien.

Selon le modèle de Harrod-Domar, la croissance sera d'autant plus forte que le taux d'épargne sera élevé et le coefficient de capital, faible. L'investissement et l'accumulation de capital sont une condition nécessaire de la croissance, et constituent les exigences initiales des autres aspects du développement. La croissance est liée à l'investissement en capital des entreprises, lui-même lié à l'épargne des ménages. En conséquence, l'État peut agir sur le niveau de croissance en favorisant l'épargne, soit par la politique des revenus (directs ou de transferts), soit par la politique fiscale et budgétaire.

Domar (1946) met en avant l'instabilité de la croissance équilibrée. Le taux de croissance est en fonction du rapport entre le taux d'épargne et le taux d'investissement. La croissance résulte donc de l'investissement, mais Harrod (1939) montre qu'il n'y a qu'un investissement qui assure l'équilibre de plein emploi, si bien que la croissance est toujours sur le fil du rasoir.

\section{Croissance et production la théorie \\ néoclassique de la croissance de Solow}

D'inspiration plutôt néoclassique, l'école de la croissance exogène, dont la théorie de Solow (1956) est une expression, montre que l'action volontaire des agents n'a pas d'influence décisive sur le rythme de la croissance. La croissance est stable et équilibrée lorsqu'il y a une « juste» répartition entre les deux facteurs de production que sont le capital et le travail. Le progrès technique est intégré à ce modèle comme une variable exogène et est défini en tant que « facteur résiduel ». Dans le modèle de Solow, il existe une 
croissance à long terme stable, dont le rythme ne dépend que de l'évolution de la population et de la technologie, et non des comportements économiques des agents. La croissance provient essentiellement de la quantité de travail (et donc de la croissance démographique) et du progrès technique, considéré comme un facteur exogène provenant de données extérieures à la croissance : le progrès scientifique.

\section{Croissance et progrès technique}

Après la généralisation de la révolution industrielle, la première moitié du XXe siècle est caractérisée par l'accélération du progrès technique et organisationnel. C'est dans ce contexte que Schumpeter (1912) fait de l'innovation le facteur explicatif de la croissance et du développement économique à long terme.

Pour Schumpeter, l'évolution ne peut venir d'une modification quantitative (p. ex., la hausse de la population ou du capital). La véritable transformation du système économique ne peut être que de nature qualitative. Si l'on définit alors le progrès technique comme l'ensemble des mutations entraînant une nouvelle manière de produire qui permet d'obtenir plus avec la même quantité de facteurs (progrès de processus) ou un nouveau produit (progrès de produit), on peut mesurer combien peuvent être étroits les liens qu'il entretient avec la croissance.

Ainsi, Schumpeter montre, au terme de son analyse, que le facteur déterminant entraînant l'évolution économique est l'innovation, essence même du capitalisme. Celle-ci est au cœur non seulement du processus de croissance, mais aussi de transformations structurelles plus importantes.

En distinguant cinq types d'innovations (produits, marchés, procédés, matières premières et organisation des entreprises), Schumpeter fait des grappes (ou vagues) d'innovation le moteur de la croissance - et l'origine des crises de mutation, par le remplacement des anciennes activités dominantes par de nouvelles industries émergentes. Dans ce processus, l'entrepreneur joue un rôle essentiel, car il est celui qui innove en insérant dans le processus économique les inventions fournies par le progrès scientifique et technique, en exploitant les perspectives de profit toujours plus élevées offertes par de nouveaux marchés, de nouvelles matières premières, de nouvelles formes d'organisation du travail, etc.

\section{Approfondissements et renouveau de la théorie de la croissance : le rôle du territoire}

\section{Théorie de la base exportatrice}

La théorie de la base exportatrice est une transposition territoriale des modèles postkeynésiens de croissance par la demande. L'hypothèse centrale de cette approche est que l'économie locale doit capter des revenus extérieurs pour se développer et que le circuit économique local démultiplie les effets de l'augmentation de ces revenus extérieurs. La croissance économique locale dépend principalement de la capacité du territoire, d'une part, à capter des revenus extérieurs par le développement des secteurs «basiques» (industrie, services aux entreprises) et, d'autre part, à limiter les fuites des revenus locaux par l'intermédiaire des activités locales «non basiques» ou «dépendantes» ou « résidentielles» (services aux personnes) (Persky, Ranney et Wiewel, 1993, cités par Diniz, Lépicier et Schmitt, 2007).

Les activités d'exportation constituent donc l'élément principal de la dynamique économique régionale, alors que la croissance des branches productrices des biens et services pour le marché local dépend des revenus distribués par le secteur d'exportation. L'approche en grappes (clusters) lui est associée et est devenue un élément incontournable des doctrines de développement régional. Selon cette approche, « la croissance et le développement dépendent de la demande externe ou des flux monétaires exogènes qui y sont liés » (Proulx, 2008a, p.12).

\section{Industries industrialisantes}

Les théories de Perroux (1961) et de Hirschman (1984) ont inspiré les stratégies des industries industrialisantes. Ces stratégies s'inspirent du modèle socialiste de développement mis en œuvre dans certains pays dès les années 1930. Selon Hirschman, le postulat de départ est que le développement est nécessairement déséquilibré : la croissance apparaît dans telle ou telle région ou encore dans tel ou tel secteur. Elle est une séquence de déséquilibres qui se propagent dans l'ensemble de l'économie à travers les complémentarités d'activités. Dans ce contexte, il convient de développer prioritairement des industries qui ont des effets d'entraînement sur l'ensemble de l'économie. Ces 
industries doivent être à forte intensité capitalistique et génératrices de goulets d'étranglement (en matière d'infrastructures, d'énergie, de matières premières).

Les industries industrialisantes sont donc celles qui, dans leur environnement local, modifient structurellement la matrice interindustrielle, transforment les fonctions de production et augmentent la productivité de l'ensemble de l'économie. Dans cette perspective, l'État joue un rôle majeur d'organisateur de polarisation des ressources en faveur de grands complexes industriels (généralement des industries lourdes). La priorité donnée à ces industries repose sur une forte intervention de l'État par la planification et par la nationalisation des entreprises.

Dans la même perspective, Perroux (1961) souligne que l'économie mondiale est marquée par une concurrence inégale entre les nations et que certaines d'entre elles ont la capacité d'orienter les flux d'échange et les structures en leur faveur (effet de domination). Il considère que le développement passe par une intervention de l'État pour favoriser l'interdépendance entre des pôles de croissance ou pôles de développement.

\section{Les sources de la croissance}

\section{dans les modèles de croissance endogène}

Au milieu des années 1980, les économistes occidentaux ont commencé à orienter leurs recherches vers un dépassement du clivage existant dans l'analyse économique entre la croissance et le développement. Au lieu de considérer que le taux de croissance est exogène ou naturel et qu'il est déterminé par la croissance démographique et un progrès technique exogène, la nouvelle théorie, dite de " croissance endogène ", tente de relier la croissance économique aux caractéristiques internes de l'économie. La nouvelle théorie de la croissance endogène a germé des contributions de Romer (1986) et de Lucas (1988), puis a supplanté l'ancienne théorie néoclassique de la croissance de Solow (1956) et de Swan (1956).

Pour les tenants des théories de la croissance endogène, la croissance résulterait de l'interaction de quatre facteurs. En investissant dans de nouveaux équipements (capital physique), l'entreprise contribue directement à la croissance (hausse de la production). Or, l'investissement induit à terme une augmentation du capital technique (par le progrès technique) et du capital humain (par la hausse du niveau d'éducation/de formation). Les investissements consentis par la collectivité (État et collectivités locales) agissent sur le capital public et sur le capital humain (éducation et santé publique). La croissance économique trouve donc sa source dans l'accumulation de différentes formes de capitaux (recherche-développement, expérience et savoirfaire, éducation et formation professionnelle, infrastructures publiques, etc.) mis en œuvre par les différents agents économiques dans un but de profit toujours plus grand.

Ainsi, la croissance est perçue comme un phénomène cumulatif: elle provoque l'accumulation du progrès technique, qui elle-même suscite la croissance. Le progrès technique serait à la fois une cause et une conséquence de la croissance.

\subsubsection{Les traits communs des dynamiques régionales du développement économique}

Sur le plan théorique, les analyses de la croissance économique des territoires reposent donc sur divers modèles qui soulignent le rôle essentiel de tel ou tel déterminant. Le modèle keynésien retient le rôle moteur du secteur exportateur et des processus d'amplification par la demande, tandis que les modèles de croissance néoclassiques mettent plutôt l'accent sur les conditions d'offre, sur l'accumulation du capital, sur l'effet de la répartition et des coûts sur les volumes ainsi que sur le progrès technique. Quant aux nouvelles théories de la croissance endogène, elles incluent le capital humain en privilégiant l'accumulation de la connaissance dans les fonctions de production.

D'après Catin (1995) et selon une approche synthétique, «la croissance régionale repose sur trois grands processus cumulatifs interconnectés, qui combinent sous différents rapports des dynamiques d'offre et de demande, internes et externes: les effets multiplicateurs, les effets de productivité, les effets de compétitivité » (p. 1). Ces trois boucles de croissance fondamentales prennent des formes différentes dans un territoire donné selon ses structures (économiques et non économiques) et agissent de manière variable au cours du temps. 
Dans les analyses de science régionale, la croissance économique a souvent été décrite comme un processus d'effets multiplicateurs, initialement générés par des activités basiques, c'est-à-dire exportatrices hors de la zone considérée. «Une croissance de la production des entreprises à activité basique occasionne des achats d'inputs à d'autres entreprises, une distribution de revenus aux facteurs qui induisent des dépenses de consommation et d'investissement, et une certaine réponse de la production locale qui relance et amplifie le processus. Ainsi, à une injection initiale exogène correspond généralement à court et à moyen terme une variation plus que proportionnelle de l'ensemble de l'activité économique régionale. En définitive, il se dégage dans une période donnée des effets multiplicateurs particuliers, caractéristiques du fonctionnement de différents circuits régionaux » (Catin, 1995, p. 3). Le principe découle de la théorie de la base exportatrice.

La croissance de la productivité est également souvent considérée comme un des principaux facteurs de la croissance économique. L'obtention des gains de productivité repose, d'une part, sur la mise en œuvre d'investissements en réponse à l'augmentation de la demande, qui conduit à des gains de productivité induite grâce à la réalisation d'économies d'échelle et, d'autre part, sur la mise en œuvre d'investissements de modernisation-rationalisation ou d'investissements d'innovation qui conduisent à des gains de productivité autonome.

Finalement, des gains de compétitivité peuvent générer deux processus de croissance : l'un reposant sur la substitution relative des importations par une production interne, l'autre sur le développe- ment des exportations. Une stratégie de compétitivité à long terme impose l'amélioration permanente de la qualité des facteurs de production. Elle est favorisée, sur le plan spatial, par l'existence d'économies d'agglomération spécifiques.

\subsubsection{Le cadre d'analyse théorique de la contribution des activités économiques au développement territorial}

Les analyses précédentes formalisent des modèles de développement et soulignent l'impact déterminant de certains facteurs dans l'atteinte d'une croissance économique. Dans ce cadre, si de manière générale « les approches de la croissance régionale épousent largement les problématiques de la croissance nationale, en les transposant dans un cadre territorial donné » (Catin, 1995, p. 2), l'espace peut, selon l'objet, être le support ou une dimension de l'analyse. Ainsi, pour les théories les plus récentes, la dynamique spatiale de l'économie est tout particulièrement prise en compte, essentiellement selon une double lecture d'endogénéité/d'exogénéité de la croissance. La spécificité spatiale du développement peut également se révéler sur le plan de la localisation et des modes d'organisation territoriale des humains et des activités ainsi de leur impact sur la croissance économique. Elle peut également se traduire en retour par l'effet de la croissance sur les disparités et les structures de production régionales.

Une manière de synthétiser les approches théoriques du développement économique en contexte territorial est de rapprocher les déterminants essentiels de la croissance tels qu'ils sont modélisés par leurs différents auteurs. 


\begin{tabular}{|c|c|c|}
\hline Théories & Contributeurs & Déterminants et leviers de la croissance proposés \\
\hline $\begin{array}{l}\text { Théories classiques du dévelop- } \\
\text { pement }\end{array}$ & $\begin{array}{l}\text { Smith } \\
\text { Ricardo } \\
\text { Malthus } \\
\text { Say }\end{array}$ & $\begin{array}{l}\text { Développement matériel de la société } \\
\text { Progrès technique } \\
\text { Croissance démographique } \\
\text { Division du travail } \\
\text { Spécialisation } \\
\text { Échanges } \\
\text { Accumulation du capital } \\
\text { Offre-demande-prix }\end{array}$ \\
\hline $\begin{array}{l}\text { Modèle keynésien de la crois- } \\
\text { sance économique }\end{array}$ & Keynes & $\begin{array}{l}\text { Politique de relance de la demande } \\
\text { Dépenses publiques } \\
\text { Investissement } \\
\text { Consommation } \\
\text { Épargne-investissement-revenu }\end{array}$ \\
\hline $\begin{array}{l}\text { Analyses postkeynésiennes de la } \\
\text { croissance }\end{array}$ & $\begin{array}{l}\text { Harrod } \\
\text { Domar }\end{array}$ & $\begin{array}{l}\text { Investissement en capital des entreprises } \\
\text { Épargne des ménages }\end{array}$ \\
\hline $\begin{array}{l}\text { Théorie néoclassique de la crois- } \\
\text { sance }\end{array}$ & Solow & $\begin{array}{l}\text { Progrès technique comme variable exogène } \\
\text { Quantité de travail } \\
\text { Croissance démographique }\end{array}$ \\
\hline Analyse schumpetérienne & Schumpeter & $\begin{array}{l}\text { Innovation à } 5 \text { niveaux : produits, marchés, procédés, matières } \\
\text { premières et organisation des entreprises } \\
\text { Entrepreneuriat }\end{array}$ \\
\hline Théorie de la base exportatrice & Keynes & Demande externe (revenus extérieurs) \\
\hline Industries industrialisantes & $\begin{array}{l}\text { Perroux } \\
\text { Hirschman }\end{array}$ & $\begin{array}{l}\text { Industries ayant la capacité d'orienter les flux d'échange } \\
\text { Polarisation }\end{array}$ \\
\hline Modèles de croissance endogène & $\begin{array}{l}\text { Romer } \\
\text { Lucas }\end{array}$ & $\begin{array}{l}\text { Interaction de différentes formes de capitaux (R-D, expérience et } \\
\text { savoir-faire, éducation et formation professionnelle, } \\
\text { infrastructures publiques, etc.) } \\
\text { Capital humain }\end{array}$ \\
\hline
\end{tabular}

Tableau 2 - Cadre d'analyse des déterminants de la croissance régionale selon quelques théories fondatrices du développement Source : compilation de l'auteur

\section{Conclusion}

Howitt (2004) a énoncé : «Une des rares leçons claires que nous a apprises la recherche sur les facteurs de prospérité à long terme est qu'il n'existe aucune solution magique, aucune explication unicausale qui débouche sur une recette de succès simple. Beaucoup de facteurs sont en jeu et ils sont largement interdépendants » (p. 13). Ne se fondant sur aucun facteur unique, le développement se présente donc tel un processus transversal. Il apparait comme multidimensionnel puisqu'aucun des facteurs pris isolément ne semble en mesure de générer le processus dans sa complexité et sa globalité.

Par ailleurs, le développement regroupe, croise et associe diverses compétences sectorielles et divers types d'acteurs. Il peut enfin être vu comme décloi- sonné, en ce sens que les limites entre les principaux facteurs contributifs apparaissent de prime abord relativement perméables (p. ex., profil des ressources humaines et équité sociale). Le processus prend davantage la forme d'un continuum intégrant des effets de cumul et d'entraînement.

En explicitant certains des paramètres critiques de cette dynamique, la présente contribution apporte un éclairage théorique et historique sur les conditions pouvant contribuer, d'une manière ou d'une autre, à un certain développement. Or, aborder la question du développement humain dans une perspective de bien commun et de durabilité nécessiterait de bien plus amples analyses. Un contexte actuel comme celui de la pandémie de COVID-19, qui amène notamment à largement relativiser les recettes garantissant la solidité de la croissance, invite, comme un contre-ballant, à réfléchir sur les priorités humaines du développement. 


\section{NOTES}

1 L'école classique en économie regroupe des économistes du XVIII siècle et du XIX ${ }^{\mathrm{e}}$ siècle. Ses membres les plus importants sont, en Grande-Bretagne, Adam Smith (1723-1790), David Ricardo (1772-1823), Thomas Malthus (1766-1834), John Stuart Mill (1806 -1873), et en France, Étienne Bonnot de Condillac (1715-1780), Anne Robert Jacques Turgot (1727-1781), Jean-Baptiste Say (1767-1832) et Frédéric Bastiat (1801-1850). [...] Nombre d'entre eux ont été des contemporains du décollage industriel anglais. (Source : Wikipédia)

\section{RÉFÉRENCES}

Assidon, E. (1992). Les théories économiques du développement. Paris, France : La Découverte.

Austruy, J., Caire, G., Leduc, G. et Lebret, L.-J. (1972). Le scandale du développement. Paris, France : M. Rivière et Cie.

Aydalot, P. (1985). Économie régionale et urbaine. Paris, France : Economica.

Brasseul, J. (2008). Introduction à l'économie du développement. Paris, France : Armand Colin.

Brunelle, C. et Shearmur, R. (2007). L'étalonnage du développement économique régional : indicateurs et mesures. Québec, QC : Institut national de la recherche scientifique - Urbanisation, Culture et Société.

Catin, M. (1995). Les mécanismes et les étapes de la croissance régionale. Région \& Développement, 1, 1-19. Repéré à http://lead.univ-tln.fr/fichiers/1995-1.pdf

Chabault, D. (2006). Les systèmes territoriaux de production : revue de littérature et approches théoriques d'un concept évolutif. Cabiers de recherche du CERMAT, 19(6). Repéré à https://www.researchgate.net/publication/255646329_Les_systemes_ territoriaux_de_production_revue_de_litterature_et_approches_theoriques_d'un_concept_evolutif

Comeliau C. (2006). La croissance ou le progrès? Croissance, décroissance, développement durable, Paris, France : Seuil.

Diniz, F., Lépicier, D. et Schmitt, B. (2007). L'intégration économique locale des entreprises et des territoires ruraux : une analyse basée sur 12 bourgs et petites villes françaises et portugaises. Revue d'économie régionale \&́urbaine, 4(novembre), 729-753. https://doi.org/10.3917/reru.074.0729

Domar, E. D. (1946). Capital Expansion, Rate of Growth and Employment. Econometrica, 14(2), 137-47.

Dugas, C. (1994). Le sous-développement régional. Dans F. Dumont, S. Langlois et Y. Martin (dir.), Traité des problèmes sociaux : quelques problèmes de population et d'aménagement (partie 1, chap. 5, p. 103-126). Québec, QC : Institut québécois de recherche sur la culture (IQRC).

Duranton, G. et Puga, D. (2005). From sectoral to functional urban specialization. Journal of Urban Economics, 57(2), 343-370. https://doi.org/10.1016/j.jue.2004.12.002

Garofoli, G. (1994). Développement endogène et rôle des acteurs locaux : un défi pour la théorie du développement. Dans IREP-D (dir.), Nowveaux dynamismes industriels et économie du développement (p. 493-499). Grenoble, France : IRED-D.

Gilly, J.-P. et Grossetti, M. (1993). Organisations, individus et territoires : le cas des systèmes locaux d'innovation. Revne d'économie régionale et urbaine, 3, 449-468.

Harrod, R. F. (1939). An Essay in Dynamic Theory. The Economic Journal, 49(193), 14-33. Repéré à http:/ /www.jstor.org/ stable/2225181

Hirschman, A. O. (1984). L'économie comme science morale et politique. Paris, France : Gallimard.

Howitt, P. (2004). Croissance endogène, productivité et politique économique : rapport de situation. Observateur international de la productivité, 8, 3-15. Repéré à https://docplayer.fr/8836447-Croissance-endogene-productivite-et-politique-economique-rapportde-situation.html

Hugon, P. (1991). L'économie du développement, le temps et l'histoire. Revue économique, 42(2), 339-364.

Repéré à https://www.persee.fr/doc/reco_0035-2764_1991_num_42_2_409282

Hummels, D. et Klenow, P. (2005, juin). The variety and quality of a nation's exports. American Economic Review, 95(3), 704-723. https://doi.org/10.1257/0002828054201396

Hunt, D. (1989). Economic Theories of Development. An Analysis of Competing Paradigms. New York, Toronto: Harvester Wheatsheaf. 
Institut de la statistique du Québec (ISQ). (2007). Produit intérieur brut régional par industrie au Québec, Repéré à www.stat.gouv.qc.ca.

Jacobs, J. (1961). The death and life of great American cities. New York, NY: Random House.

Jean, B. (2008). Le développement territorial : une discipline émergente. Dans G. Massicotte (dir.), Sciences du territoire : perspectives québécoises (p. 283-313). Québec, QC : Presses de l'Université du Québec.

Kaldor, N. (1957). A model of economic growth. The Economic Journal, 67(268), 591-624. https://doi.org/10.2307/2227704

Keynes, J.M. (1936). The General Theory of Employment, Interest, and Money. Cambridge, GB: Cambridge University Press, for the Royal Economic Society Cambridge University Press

Klein, J.-L. (2008). Territoire et développement : du local à la solidarité interterritoriale. Dans G. Massicotte (dir.), Sciences du territoire : perspectives québécoises (p. 315-333). Québec, QC : Presses de l’Université du Québec.

Klibanoff, P. et Morduch, J. (1995). Decentralization, externalities, and efficiency. Review of Economic Studies, 62(2), 223-247. https://doi.org/10.2307/2297803

Lucas, R. E. Jr. (1988). On the Mechanics of Economic Development. Journal of Monetary Economics, 22(January), 3-42.

Malaga, K. (2009). À propos des quelques dilemmes de la théorie de croissance économique et d'économie. Romanian Journal of Economics, 29(2), 38-54. Repéré à http://revecon.ro/articles/2009-2/2009-2-3.pdf

Martens, A. (2005). Développement et économie du développement. Montréal, QC : Université de Montréal, Département de sciences économiques.

Myrda, G. (1957). Economic Theory and Underdeveloped Regions. Londres, GB: Duckworth.

Perroux, F. (1955). Note sur la notion de pôle de croissance. Économie appliquée, 8, 307-320.

Perroux, F. (1961). L'économie du XXe siècle. Grenoble, France : Presses universitaires de Grenoble.

Persky, J., Ranney, D. et Wiewel, W. (1993). Import substitution and local economic development. Economic Development Quarterly, 7 , 18-29. https://doi.org/10.1177\%2F089124249300700103

Prager, J.-C. et Thisse, J.-F. (2009). Les enjeux géographiques du développement économique. Paris, France : Agence française de développement, Département de la recherche.

Programme des Nations unies pour le développement (PNUD) (1991). Rapport mondial sur le développement bumain. Paris, France : Economica.

Proulx, M.-U. (2008a). Économie des territoires. Québec, QC : Presses de l’Université du Québec.

Proulx, M.-U. (2008b). Sciences du territoire : territoires de gestion et territoires d'émergence. Québec, QC : Presses du l’Université du Québec.

Rist, G. (2007). L'environnement ou la nouvelle nature du « développement ». Dans G. Rist (dir.), Le développement: bistoire d’une croyance occidentale (chap. 10, p. 299-343). Paris, France : Presses de Sciences Po.

Rist, G. (2015). Le développement : Histoire d'une croyance occidentale. Paris, France : Presses de Sciences Po.

Romer, P. M. (1986). Increasing Returns and Long-Run Growth. Journal of Political Economy, 94(October), 1002-1037.

Rostow, W. W. (1962). Les étapes de la croissance économique. Revne économique, 16(4), 629-630.

Schott, P. K. (2004). Across-product versus within-product specialization in international trade. Journal of Economics, 119(2), 647-678. https://doi.org/10.1162/0033553041382201

Schumpeter J. (1912). The Theory of Economic Development. Cambridge, GB: Harvard University Press.

Smith, A. (1776). Recherche sur la nature et les causes de la richesse des nations. Londres, GB : Strahan and Cadell Eds.

Solow R. (1956). A contribution to the Theory of Economic Growth. Quaterly Journal of Economics, 70(1), 65-94.

Swan, T. W. (1956). Economic Growth and Capital Accumulation. Economic Record, 32(november), 334-61.

Thant, U. (1962). Décennie des Nations Unies pour le développement (ONU).

Repéré à https: / / research.un.org/c.php?g $=856353 \& p=6278592$ 
Theys, J. (2002). L'approche territoriale du " développement durable ", condition d'une prise en compte de sa dimension sociale, Développement durable et territoires [En ligne]. https://doi.org/10.4000/developpementdurable.1475

Vivien, F. (2003). Jalons pour une histoire de la notion de développement durable. Mondes en développement, 121(1), 1-21. https://doi.org/10.3917/med.121.0001 